\title{
Explicit construction of the classical BRST charge for nonlinear algebras
}

\author{
A.V.Bratchikov \\ Kuban State Technological University, \\ 2 Moskovskaya Street, Krasnodar, 350072, Russia
}

March, 2011

\begin{abstract}
We give an explicit formula for the Becchi-Rouet-Stora-Tyutin (BRST) charge associated with Poisson superalgebras. To this end, we split the fundamental equation for the BRST charge into a pair of equations such that one of them is equivalent to the original one. We find the general solution to this equation. The solution possesses a graphical representation in terms of diagrams.
\end{abstract}

\section{Introduction}

The BRST symmetry [1, 2] plays an important role in quantization of gauge theories [3, 4. It is generated by the BRST charge. If the quantum BRST charge exists it is essentially determined by the corresponding classical one.

The classical BRST charge is represented by a power series in ghosts. The first two terms of the series are well known in the general case. When constraints form a Lie algebra, these terms reproduce an exact BRST charge.

The fundamental equation for the BRST charge is equivalent to a system of recurrent equations. In the case of general Poisson algebras there exists an algorithm for the construction of a solution to these equations [5].

For some classes of quadratically nonlinear algebras the classical BRST charge was found in [6, 7]. Construction of the BRST charge for some boson Poisson algebras was investigated in [8]. In the case of general quadratically 
nonlinear algebras the expression for the third order contribution in the ghost fields to the BRST charge was found. In 99, the classical BRST charge for quadratically nonlinear superalgebras was discussed. For some classes of superalgebras the BRST charge was constructed up to the fourth order in the ghost fields.

In this paper we derive an explicit expression of the classical BRST charge for nonlinear Poisson superalgebras. We show that the system of equations for the classical BRST charge is equivalent to a smaller subsystem. Then we find the general solution to the subsystem. Expanding the solution in powers of the ghost fields one can find the BRST charge in an arbitrary order.

The paper is organized as follows. In section 2, we introduce notations and represent the master equation for the BRST charge in the form which is convenient for our purposes. In section 3, we obtain an explicit expression for the classical BRST charge. We show that the expression possesses a graphical representation in terms of diagrams.

In what follows Grassman parity and ghost number of a function $A$ are denoted by $\epsilon(A)$ and $\operatorname{gh}(A)$, respectively.

\section{Structure of the master equation for the BRST charge}

Let $G_{\alpha}, \alpha=1, \ldots, J$, be the first class constraints which satisfy the following Poisson brackets

$$
\left\{G_{\alpha}, G_{\beta}\right\}=F_{\alpha \beta}(G)
$$

where $F_{\alpha \beta}(G)$ is a polynomial in the $G$ 's such that $F_{\alpha \beta}(0)=0$. The constraints are supposed to be independent and of definite Grassmann parity $\epsilon_{\alpha}, \epsilon\left(G_{\alpha}\right)=\epsilon_{\alpha}$.

Following the BRST method the ghost pair $\left(\mathcal{P}_{\alpha}, c^{\alpha}\right)$ is introduced for each constraint $G_{\alpha}$ :

$$
\begin{gathered}
\left\{\mathcal{P}_{\alpha}, c^{\beta},\right\}=\delta_{\beta}^{\alpha}, \quad\left\{G_{\alpha}, c^{\beta}\right\}=\left\{\mathcal{P}_{\alpha}, G_{\beta},\right\}=0 \\
\epsilon\left(\mathcal{P}_{\alpha}\right)=\epsilon\left(c^{\alpha}\right)=\epsilon\left(G_{\alpha}\right)+1 \\
-\operatorname{gh}\left(\mathcal{P}_{\alpha}\right)=\operatorname{gh}\left(c^{\alpha}\right)=1
\end{gathered}
$$


Let $\mathcal{M}$ be the set of variables $\left(G^{\alpha}, \mathcal{P}_{\beta}, c^{\gamma}\right)$, and let $\mathcal{V}=R[[\mathcal{M}]]$ be the ring of formal power series in the variables $\mathcal{M}$.

The BRST charge $\Omega \in \mathcal{V}$ is defined as a solution to the equation

$$
\{\Omega, \Omega\}=0, \quad \epsilon(\Omega)=1, \quad \operatorname{gh}(\Omega)=1,
$$

and the boundary conditions

$$
\left.\frac{\partial \Omega}{\partial c^{\alpha}}\right|_{c=0}=G_{\alpha} .
$$

These equations are consistent [10, 11]. One can write

$$
\Omega=G_{\alpha} c^{\alpha}+M,
$$

where

$$
M=\sum_{n=2}^{J} \Omega^{(n)}, \quad \Omega^{(n)} \sim \mathcal{P}^{n-1} c^{n} .
$$

Substituting (3) into (11) one obtains

$$
\delta M+\frac{1}{2} F+A M+\frac{1}{2}\{M, M\}=0,
$$

where

$$
\delta=G_{\alpha} \frac{\partial_{l}}{\partial \mathcal{P}_{\alpha}}, \quad F=c^{\alpha} F_{\alpha \beta}(G) c^{\beta}, \quad A=c^{\alpha}\left\{J_{\alpha}, .\right\} .
$$

Let $N$ be the counting operator

$$
N=G_{\alpha} \frac{\partial_{l}}{\partial G_{\alpha}}+\mathcal{P}_{\alpha} \frac{\partial_{l}}{\partial \mathcal{P}_{\alpha}} .
$$

The space $\mathcal{V}$ splits as

$$
\mathcal{V}=\bigoplus_{n \geq 0} \mathcal{V}_{n}
$$

with $N X=n X$ for $X \in \mathcal{V}_{n}$. One easily verifies that

$$
N=\delta \sigma+\sigma \delta, \quad \sigma=\mathcal{P}_{\alpha} \frac{\partial_{l}}{\partial G_{\alpha}}
$$




$$
N \delta=\delta N, \quad N \sigma=\sigma N .
$$

We define $N^{+}: \mathcal{V} \rightarrow \mathcal{V}$ by

$$
N^{+} X= \begin{cases}\frac{1}{n} X, & X \in \mathcal{V}_{n}, \quad n>0 \\ 0, & X \in \mathcal{V}_{0} .\end{cases}
$$

Then $\delta^{+}=\sigma N^{+}$is a generalized inverse of $\delta$ :

$$
\delta \delta^{+} \delta=\delta, \quad \delta^{+} \delta \delta^{+}=\delta^{+} .
$$

Let $\langle.,\rangle:. \mathcal{V}^{2} \rightarrow \mathcal{V}$ be defined by

$$
\left\langle X_{1}, X_{2}\right\rangle=-\frac{1}{2}\left(I+\delta^{+} A\right)^{-1} \delta^{+}\left(\left\{X_{1}, X_{2}\right\}+\left\{X_{2}, X_{1}\right\}\right),
$$

where $I$ is the identity map, and

$$
\left(I+\delta^{+} A\right)^{-1}=\sum_{m \geq 0}(-1)^{m}\left(\delta^{+} A\right)^{m}
$$

Lemma 1. Eq. (4) is equivalent to

$$
M=M_{0}+\frac{1}{2}\langle M, M\rangle,
$$

where

$$
M_{0}=\left(I+\delta^{+} A\right)^{-1}\left(Y-\frac{1}{2} \delta^{+} F\right)
$$

and $Y \in \mathcal{V}$ is an arbitrary cocycle, $\delta Y=0$, subject only to the restrictions

$$
\begin{array}{cc}
\epsilon(Y)=1, \quad & \operatorname{gh}(Y)=1, \\
Y=\sum_{n=2}^{J} Y^{(n)}, \quad Y^{(n)} \sim \mathcal{P}^{n-1} c^{n} .
\end{array}
$$

Proof. In accordance with the decomposition

$$
\mathcal{V}=\mathcal{V}_{1} \oplus \mathcal{V}_{2}
$$


where

$$
\mathcal{V}_{1}=P \mathcal{V}, \quad \mathcal{V}_{2}=(I-P) \mathcal{V}, \quad P=\delta \delta^{+},
$$

Eq.(4) splits as

$$
\begin{gathered}
\delta M+\delta \delta^{+} D=0 \\
\left(I-\delta \delta^{+}\right) D=0
\end{gathered}
$$

where

$$
D=\frac{1}{2} F+A M+\frac{1}{2}\{M, M\} .
$$

From (8) it follows that

$$
M=Y-\delta^{+} D
$$

where the cocycle $Y \in \mathcal{V}$ satisfies (17). Eq. (10) can be rewritten in the form (5). One can show that $Y=\delta W, W \in \mathcal{V}[11$.

Eq. (5) can be iteratively solved as:

$$
M=M_{0}+\frac{1}{2}\left\langle M_{0}, M_{0}\right\rangle+\ldots
$$

Using (11), we can write

$$
\Omega^{(n)}=\delta W^{(n)}+\widetilde{\Omega}^{(n)}\left(Y^{(2)}, Y^{(3)}, \ldots, Y^{(n-1)}\right), \quad n \geq 2,
$$

where $\delta W^{(n)}=Y^{(n)}$. The arbitrariness of the solution for $\Omega^{(n)}$ is described by the transformation [12

$$
\Omega^{(n)} \rightarrow \Omega^{(n)}+\delta Z^{(n)}, \quad Z \in \mathcal{V} .
$$

This transformation is absorbed into a transitive group transformation of the coboundary $\delta W^{(n)}$ :

$$
\delta W^{(n)} \rightarrow \delta W^{(n)}+\delta Z^{(n)} .
$$

Therefore, $M$ (11) is the general solution to eq. (44). It follows that Eq. (4) is equivalent to (5). Eq. (9) can be omitted.

In the next section, we obtain an explicit solution to Eq. (5) ). 


\section{Explicit expression for the BRST charge}

To solve eq. (5) we introduce the functions

$$
\langle\ldots\rangle: \mathcal{V}^{m} \rightarrow \mathcal{V}, \quad m=1,2, \ldots,
$$

which recursively defined by

$$
\langle X\rangle=X
$$

$\left\langle X_{1}, \ldots, X_{m}\right\rangle=\frac{1}{2} \sum_{r=1}^{m-1} \sum_{1 \leq i_{1}<\ldots<i_{r} \leq m}\left\langle\left\langle X_{i_{1}}, \ldots, X_{i_{r}}\right\rangle,\left\langle X_{1}, \ldots, \widehat{X}_{i_{1}}, \ldots, \widehat{X}_{i_{r}}, \ldots, X_{m}\right\rangle\right\rangle(1$

if $m=2,3, \ldots$, where $\widehat{X}$ means that $X$ is omitted.

The following lemma is easily proved by induction.

Lemma 2. $\left\langle X_{1}, \ldots, X_{m}\right\rangle$ is an $m$-linear symmetric function.

For $m \geq 2,1 \leq i, j \leq m$, let

$$
P_{i j}^{m}: \mathcal{V}^{m} \rightarrow \mathcal{V}^{m-1}
$$

be defined by

$$
P_{i j}^{m}\left(X_{1}, \ldots, X_{m}\right)=\left(\left\langle X_{i}, X_{j}\right\rangle, X_{1}, \ldots, \widehat{X}_{i}, \ldots, \widehat{X}_{j}, \ldots, X_{m}\right) .
$$

If $X \in \mathcal{V}$ is given by

$$
X=P_{12}^{2} P_{i_{m-2} j_{m-2}}^{3} \ldots P_{i_{2} j_{2}}^{m-1} P_{i_{1} j_{1}}^{m}\left(X_{1}, \ldots, X_{m}\right)
$$

for some $\left(i_{1} j_{1}\right), \ldots,\left(i_{m-2} j_{m-2}\right)$, we say that $X$ is a descendant of $\left(X_{1}, \ldots, X_{m}\right)$. A descendant of $X \in \mathcal{V}$ is defined as $X$.

The function $\left\langle X_{1}, \ldots, X_{m}\right\rangle$ can be described by diagrams. In these diagrams an element of $\mathcal{V}$ is represented by the line segment - . A product $\left(X_{i}, X_{j}\right) \rightarrow\left\langle X_{i}, X_{j}\right\rangle$ is represented by the vertex joining the line segments for $X_{i}, X_{j}$ and $\left\langle X_{i}, X_{j}\right\rangle$ (see figure 1).

Lemma 3. 13. The function $\left\langle X_{1}, \ldots, X_{m}\right\rangle$ equals the sum of all the descendants of $\left(X_{1}, \ldots, X_{m}\right)$.

For example,

$$
\left\langle X_{1}, X_{2}, X_{3}\right\rangle=\left\langle\left\langle X_{1}, X_{2}\right\rangle, X_{3}\right\rangle+\left\langle\left\langle X_{1}, X_{3}\right\rangle, X_{2}\right\rangle+\left\langle\left\langle X_{2}, X_{3}\right\rangle, X_{1}\right\rangle .
$$




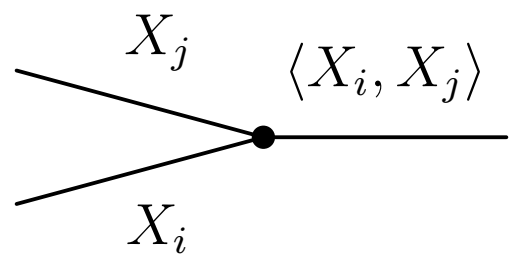

Figure 1: $\left(X_{i}, X_{j}\right) \rightarrow\left\langle X_{i}, X_{j}\right\rangle$.

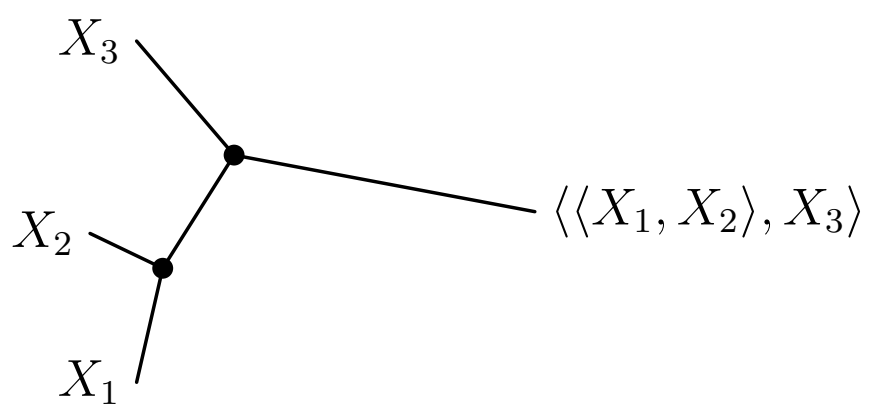

Figure 2: Diagram for $\left\langle\left\langle X_{1}, X_{2}\right\rangle, X_{3}\right\rangle$.

In figure 2, we show the diagram for $\left\langle\left\langle X_{1}, X_{2}\right\rangle, X_{3}\right\rangle$.

Lemma 4. [13] A solution to Eq.(5) is given by

$$
M=\left\langle e^{M_{0}}\right\rangle,
$$

where

$$
\left\langle e^{M_{0}}\right\rangle=\sum_{m \geq 0} \frac{1}{m !}\left\langle M_{0}^{m}\right\rangle, \quad\left\langle M_{0}^{r}\right\rangle=\langle\underbrace{M_{0}, \ldots, M_{0}}_{r \text { times }}\rangle, \quad\left\langle M_{0}^{0}\right\rangle=0 .
$$

Our previous results lead to the following theorem.

Theorem. The general solution to Eqs. (1), (2) is given by

$$
\Omega=G_{\alpha} c^{\alpha}+\sum_{m \geq 1} \frac{1}{m !}\left\langle M_{0}^{m}\right\rangle .
$$


From (6) it follows immediately that $M_{0}=O\left(c^{2}\right)$. Using (12) and the induction method, one can show that

$$
\left\langle M_{0}^{m}\right\rangle=O\left(c^{m+1}\right)
$$

Hence, in the case of bosonic constraints, $\epsilon\left(G_{\alpha}\right)=0, \epsilon\left(c^{\alpha}\right)=1$, eq. (14) takes the form

$$
\Omega=G_{\alpha} c^{\alpha}+\sum_{m=1}^{J-1} \frac{1}{m !}\left\langle M_{0}^{m}\right\rangle .
$$

For example, using lemma 1, we get

$$
\Omega=G_{\alpha} c^{\alpha}+\sum_{m=1}^{5} \frac{1}{m !}\left\langle M_{0}^{m}\right\rangle+O\left(c^{7}\right),
$$

where

$$
\begin{aligned}
\frac{1}{3 !}\left\langle M_{0}^{3}\right\rangle= & \frac{1}{2}\left\langle\left\langle M_{0}, M_{0}\right\rangle, M_{0}\right\rangle, \\
\frac{1}{4 !}\left\langle M_{0}^{4}\right\rangle= & \frac{1}{2}\left\langle\left\langle\left\langle M_{0}, M_{0}\right\rangle, M_{0}\right\rangle, M_{0}\right\rangle+\frac{1}{8}\left\langle\left\langle M_{0}, M_{0}\right\rangle,\left\langle M_{0}, M_{0}\right\rangle\right\rangle, \\
\frac{1}{5 !}\left\langle M_{0}^{5}\right\rangle= & \frac{1}{2}\left\langle\left\langle\left\langle\left\langle M_{0}, M_{0}\right\rangle, M_{0}\right\rangle, M_{0}\right\rangle, M_{0}\right\rangle+\frac{1}{4}\left\langle\left\langle\left\langle M_{0}, M_{0}\right\rangle, M_{0}\right\rangle,\left\langle M_{0}, M_{0}\right\rangle\right\rangle+ \\
& +\frac{1}{8}\left\langle\left\langle\left\langle M_{0}, M_{0}\right\rangle,\left\langle M_{0}, M_{0}\right\rangle\right\rangle, M_{0}\right\rangle .
\end{aligned}
$$

\section{References}

[1] C. Becchi, A. Rouet, R. Stora, Commun. Math. Phys. 42, 127 (1975)

[2] I. V. Tyutin, Preprint No. 39, Lebedev Physics Institute, (Moscow, Russia, 1975)

[3] D. M. Gitman, I. V. Tyutin, Quantization of fields with constraints, (Springer-Verlag, 1990) 
[4] M. Henneaux, C. Teitelboim, Quantization of gauge systems, (Prinston Univ. Press, 1992)

[5] E. S. Fradkin, T. E. Fradkina, Phys. Lett. 72B, 343 (1978)

[6] K. Schoutens, A. Sevrin, P. van Nieuwenhuizen, Commun. Math. Phys. 124, 87 (1989)

[7] A. Dresse, M. Henneaux, J. Math. Phys. 35, 1334 (1994)

[8] I. L. Buchbinder, P. M. Lavrov, J. Math. Phys. 48, 082306 (2007)

[9] M. Asorey, P. M. Lavrov, O. V. Radchenko, A. Sugamoto, Int. J. Mod. Phys. A 24, 5033 (2009)

[10] M. Henneaux, Phys.Rep. 126, 1 (1985)

[11] I. A. Batalin, P. M. Lavrov, I. V. Tyutin, J. Math. Phys. 31, 6 (1990)

[12] I. A. Batalin, I. V. Tyutin, Int. J. Mod. Phys. A 6, 3255 (1991)

[13] A. V. Bratchikov, Solving field equations in spinor electrodynamics, arXiv:0910.0621[hep-th]. 